Check for updates

Cite this: Phys. Chem. Chem. Phys., 2019, 21, 7907

\title{
Structure dependent optoelectronic properties of monolayer antimonene, bismuthene and their binary compound
}

\author{
D. Kecik, ${ }^{a}$ V. O. Özçelik, (DD ${ }^{b}$ E. Durgun (D) ${ }^{* c}$ and S. Ciraci*a
}

\begin{abstract}
Two-dimensional (2D) antimonene, bismuthene, and their binary compound 2D BiSb possess high spin-orbit coupling (SOC) and potential topological insulator properties upon engineering their structural and chemical properties. Based on many-body first-principles calculations, we show that these materials can exhibit isotropic or anisotropic optoelectronic properties depending on their geometry, i.e. buckled (hb) or asymmetrical washboard (aw) phases. SOC significantly alters their optoelectronic properties, which is predominantly evident in 2D bismuthene. hb-antimonene absorbs light in the visible and partially in the ultraviolet regimes, while the absorption band edge for aw-antimonene, hb- and aw-bismuthene is set at the infrared region, absorption being spread as a broadband optical response through the spectral range. An exciton binding with $0.18 \mathrm{eV}$ energy is detected for hb-bismuthene. Due to their broadband optical response, antimonene, bismuthene, and their binary compound offer possibilities towards applications as 2D materials in solar cells, light-emitting devices, photodetectors and light modulation.
\end{abstract}

Received 29th November 2018, Accepted 14th March 2019

DOI: $10.1039 / c 8 c p 07344 a$

rsc.li/pccp that can offer promising features suitable for $2 \mathrm{D}$ electronics and optics. $^{25}$

Among the pnictogens, antimonene and bismuthene gained particular importance for the following reasons: (i) the energy range of antimonene's fundamental band gap, which is $\sim 0.3-2.2 \mathrm{eV}$, keeps the promise of potential applications in $2 \mathrm{D}$ electronic and optical nanodevices. ${ }^{21,26-31}$ (ii) Buckled antimonene nanoribbons are semiconductors, and their band gaps vary with their chirality and width. ${ }^{32,33}$ Similarly, the band gap of nanoribbons with the aw structure vary also with their width, offering tunability of the band gap. (iii) $\mathrm{Sb}$ and Bi elements can form a stable single-layer (SL) binary compound, which is a semiconductor with a fundamental band gap different from the parent constituents. Additionally, this compound exhibits topologically non-trivial behavior under specific conditions. ${ }^{34,35}$ (iv) Bi possesses considerable spin-orbit coupling. While threedimensional (3D) hexagonal Bi crystal with a small direct band gap and inverted indirect band gap is a semimetal, it becomes a topological insulator under strain. ${ }^{36}$ Monolayer hb-bismuthene is topologically non-trivial based on the density functional theory (DFT)-spin-orbit coupling (SOC) calculations. ${ }^{37}$ Also, hb-Bi placed on a Si substrate was shown to be topologically non-trivial. DFT-SOC calculations of aw-Bi indicated a trivial topological order. ${ }^{37}$ (v) Finally, significant progress has been made towards the synthesis of SL antimonene and bismuthene structures. Ultrathin films of antimonene have been synthesized using methods such as mechanical/liquid-phase exfoliation or epitaxial growth. ${ }^{38-40} 2 \mathrm{D}$ bismuthene was realized on a 
SiC substrate, where wide fundamental band gaps and significant SOC effects were observed. ${ }^{41}$

Building on the aforementioned properties of SL Sb and Bi, it is possible to form a compound of these two elements in order to achieve superior optoelectronic properties, and obtain new target materials by inverse design; a concept that has been gaining growing attention. ${ }^{42}$ In fact, BiSb is already present in nature in a layered bulk crystal form, from which single layers of BiSb can be exfoliated, according to the previous theoretical studies. ${ }^{43,44} 2 \mathrm{D} \mathrm{BiSb}$ is particularly interesting due to the fact that both SL bismuthene and antimonene show high SOC, a precondition of stable topological insulators. ${ }^{35}$ Hence it is crucial to have a complete understanding of the electronic and optical properties of such compounds for future applications.

\section{Current state of the problem}

The optical properties of antimonene and bismuthene have been studied, with a predominant emphasis on the former. ${ }^{25,28,30,31,45,46}$ Alongside the $\mathrm{DFT}^{28}$ and hybrid functional (HSE) calculations (with random-phase approximation (RPA) employed subsequently) of hb- and aw-antimonene, ${ }^{30}$ Shu et $a{ }^{31}{ }^{31}$ studied the optical properties of hb-antimonene using a many-body approach, under the effects of electric field and strain. They reported that a strong electric field closed the band gap of aw-Sb and an increasing strain level resulted in a red-shift of the optical spectra, with enhanced optical absorption within 1.2-2.2 eV. They found the $G_{0} W_{0}$ band gap to be indirect and $2.25 \mathrm{eV}$, and an exciton binding energy of $0.73 \mathrm{eV}$ was calculated. The optical properties of monolayer hb-Sb can also be tuned via surface charge doping by $\mathrm{p} / \mathrm{n}$ type surface dopants, which revealed enhanced absorption of light especially in the early photon energy regime. ${ }^{45}$ In a recent study employing the many-body Green's function approach, the fundamental band gap of hb-Sb was predicted as $2.38 \mathrm{eV}$. Moreover, the optical and luminescence band gaps of SL hb-Sb were found to be $2.3 / 1.5 \mathrm{eV}$, resulting in an exciton binding energy of $0.8 \mathrm{eV}{ }^{46}$ Promising indications of antimonene's broadband use as a photodetector or optical modulator have been shown recently. ${ }^{47,48}$

Bismuthene's optical properties have not been vastly investigated yet, with only a few recent examples of electric field applications or its potential as a photonic-based device, ${ }^{45,49-51}$ hence the light-matter interaction within 2D bismuthene remains an area of interest. While the dynamical stability, electronic structure and topological features of SL BiSb binary compounds have been studied actively, ${ }^{34,35,52-56}$ very little is known about their optical properties. Stable binary compounds of 2D BiSb were systematically investigated from first-principles by Xiao et al., ${ }^{52}$ who found few stable aw-BiSb alloy structures, one of them having a direct HSE band gap of $0.43 \mathrm{eV}$. Regarding the effects of strain on the electronic band structure and stability of SL BiSb, the compound turned out to be a semiconductor with a direct band gap as well, reducing under strain, and undergoing a direct-indirect-metal transition when compressive strain is applied. ${ }^{53}$ With the inclusion of SOC in SL hb-BiSb, a Rashba spin-splitting of $13 \mathrm{meV}$ near the Fermi level was observed, which can be tuned by in-plane biaxial strain, making this material a candidate for efficient spin field-effect transistors, optoelectronics, and spintronic devices. ${ }^{34}$ The same material was predicted to be a quantum spin Hall insulator under biaxial strain. ${ }^{54}$

In this paper, by performing first-principles calculations and incorporating many-body interactions, we show that SL hb and aw phases of $\mathrm{Sb}$ and $\mathrm{Bi}$, as well as $2 \mathrm{D}$ BiSb, display optical activities that span the infrared (IR), visible and ultraviolet (UV) light regimes partially or entirely, and exhibit either an isotropic or anisotropic optical response depending on their geometrical phases. We present a complete analysis of the optoelectronic properties of $2 \mathrm{D} \mathrm{Sb}$ and $\mathrm{Bi}$ which also shed light on the possible functionalities of their compounds. We calculate these properties using the HSE-SOC + RPA approach, i.e. employing RPA on top of the HSE ground state calculations with spin-orbit coupling effects taken into account. Moreover, we report the HSE-SOC and $G_{0} W_{0}$ band gaps of all pristine structures. Finally, we investigate the excitonic effects within the optical properties of two representative materials, hb-Sb and hb-Bi.

\section{Methodology}

The ground-state electronic structure calculations were performed for the $2 \mathrm{D} \mathrm{hb}$ - and aw-structures of $\mathrm{Sb}$ and $\mathrm{Bi}$, using spinpolarized DFT. The projector-augmented-wave potentials (PAW) formalism $^{57-59}$ implemented in the VASP $\operatorname{code}^{60,61}$ was used. The electron exchange and correlation (XC) potential was described by the Perdew-Burke-Ernzerhof (PBE) form within the generalized gradient approximation (GGA), with XC correlation potential parameters tuned according to GW parameters. ${ }^{62}$ The plane-wave basis set was used with an energy cutoff of 520 $\mathrm{eV}$ for all calculations. Atomic positions were optimized using the conjugate gradient method by minimizing the total energy and atomic forces. The energy difference between the sequential steps was set to $10^{-6} \mathrm{eV}$ for convergence. The maximum allowed force on each atom and Gaussian smearing factor were taken as $0.05 \mathrm{eV}^{-1}$ and $0.05 \mathrm{eV}$, respectively. A $\Gamma$-centered $127 \times 127 \times 1$ grid was employed for the Brillouin zone (BZ) integration of the primitive unit cells. In order to avoid spurious interactions between the periodic images, a supercell with minimum $\sim 20 \AA$ vacuum space was adopted.

Subsequent to the lattice optimizations, hybrid functionals (HSE06) ${ }^{63-65}$ and the quasiparticle (QP) non-self consistent $G_{0} W_{0}$ approach $\left[(\mathrm{QP}) G_{0} W_{0}\right],{ }^{66-68}$ where $G$ and $W$ are iterated once, were used (without further ionic relaxation) to obtain the corrected band structures and optical spectra of hb-Sb, hb-Bi, $\mathrm{aw}-\mathrm{Sb}$, and aw-Bi. The updated wavefunctions given by HSE are used as a basis for the (QP) $G_{0} W_{0}$ calculations. RPA ${ }^{69,70}$ was employed on top of the HSE and $G_{0} W_{0}$ approaches, using a total of 192 (valence and conduction) bands. Due to the heavy computational workload, the $k$-mesh was reduced to $19 \times 19 \times$ 1 and $13 \times 13 \times 1$ for hb- and aw-structures, respectively, while calculating the quasiparticle band energies. The screening was evaluated at 240 frequency points for the majority of the (QP) $G_{0} W_{0}$ 
calculations. The plane-wave cutoff was decreased to $350 \mathrm{eV}$ and the basis set for the response functions was set to $240 \mathrm{eV}$ for the $G_{0} W_{0}$ calculations. Local field effects were accounted for both at the levels of Hartree and exchange-correlation potential. The SOC effects were included self-consistently by rotating all spins with respect to the spin quantization axis $(0,0,1)$, both at the levels of PBE and HSE. ${ }^{71}$ For all structures, the SOC effects were incorporated continuously, during the steps of lattice optimization and computation of the RPA spectra.

Regarding the optical response beyond the independentparticle approach, the electron-hole interactions were taken into account by solving the Bethe-Salpeter equation (BSE) ${ }^{72-74}$ within the Tamm-Dancoff approximation, using the same $k$-point sampling, energy cutoff value and number of bands as in the $(\mathrm{QP}) G_{0} W_{0}$ calculation. ${ }^{75}$ The excitonic effects were considered by including the eight highest occupied valence and ten lowest unoccupied conduction bands. Further to the main optical response function, the frequency dependent imaginary dielectric function $\varepsilon_{2}(\omega)$, layer dependent absorbance $[A(\omega)]$ can be obtained through the relation: $A(\omega)=\omega L \varepsilon_{2}(\omega) / c, L$ being the distance between two periodic images of the $2 \mathrm{D}$ material and $c$ the speed of light.

\section{D antimonene and bismuthene}

\section{Atomic and electronic structures}

In recent theoretical studies, ${ }^{20,23,76,77}$ three stable phases, namely hexagonal buckled (hb- or $\beta$-phase), asymmetric washboard (aw- or $\alpha$-phase), and square-octagon (so-), of 2D antimonene and bismuthene were suggested. On the other hand, the symmetric washboard (sw) structure, which is a stable phase for phosphorene and arsenene, is destabilized for antimonene and bismuthene. In the present study, we consider only the hb- and aw-phases as described in Fig. 1, and calculate their optimized atomic structure and self-consistent field electronic band structure for the sake of completeness, which constitute the basis for the present study of the optical properties.

Optimized structural parameters, such as 2D lattice constants $a$ and $b$, bond lengths $d_{1}$ and $d_{2}$, and buckling parameter $\Delta$, are presented in Table 1 . Electronic energy structures of these 2D monolayers are calculated firstly within PBE-SOC, and subsequently corrected by HSE-SOC and $G_{0} W_{0}$. The standard DFT calculations incorporating SOC effects are performed only in order to provide a basis for band gap comparisons, and are out of scope in terms of the optical properties. The calculated fundamental band gaps together with the results of earlier calculations are also listed in Table 1. According to the HSESOC calculations, hb-Sb is an indirect band gap semiconductor with a band gap of $1.12 \mathrm{eV}$, in comparison to the $0.78 \mathrm{eV}$ indirect PBE-SOC gap. This gap is predicted as $1.57 \mathrm{eV}$ from the $G_{0} W_{0}$ calculations. The direct fundamental band gap of aw-Sb is relatively smaller and calculated as $0.16,0.23$ and $0.37 \mathrm{eV}$ from the PBE-SOC, HSE-SOC and $G_{0} W_{0}$ calculations, respectively. Using the HSE-SOC (PBE-SOC) approach, we predict the fundamental (indirect) band gap of hb-Bi and aw-Bi structures

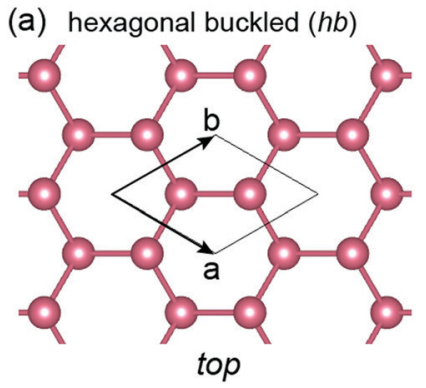

(b) asymm. washboard (aw)
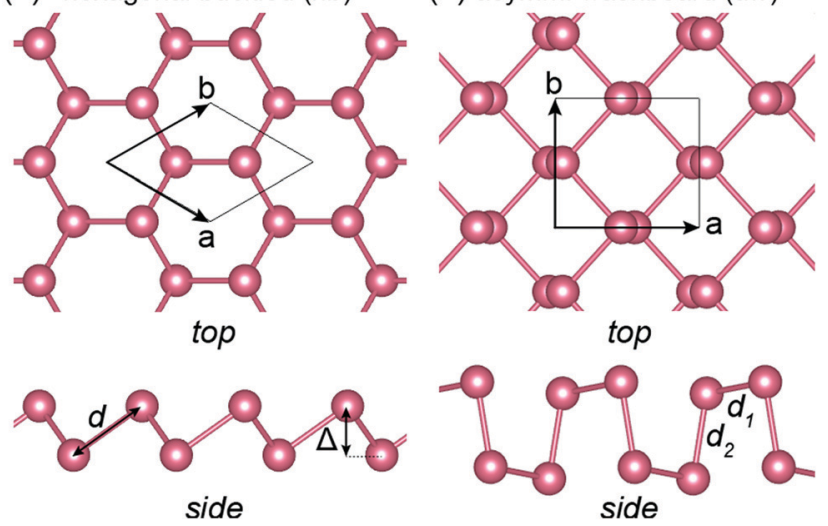

Fig. 1 (a) Top and side views of hb- (or $\beta$-phase) and (b) aw- (or $\alpha$-phase) structures of antimonene and bismuthene. The primitive unit cell vectors are designated by the lattice constants $a$ and $b$. The buckling parameter $\Delta$ and bond length $d$ of the hb-structure, and two different bond lengths $d_{1}$ and $d_{2}$ of the aw-structure, are also shown.

as $0.45(0.33)$ and $0.25(0.14) \mathrm{eV}$, respectively. These values increase to 0.92 and $0.35 \mathrm{eV}$ as a result of the $G_{0} W_{0}$ calculations. In Fig. 2, the band structures of hb-Sb, aw-Sb, hb-Bi and aw-Bi calculated from HSE-SOC are presented. The fundamental band gaps calculated from $G_{0} W_{0}$ are also shaded in the same figure.

\section{Optical absorption spectra}

SL antimonene and bismuthene structures' electronic band gaps lie within a certain range, as discussed in the previous section. This diversity in between the IR and visible regimes is quite promising in terms of their optical properties. Topological features displayed by a few of the phases promise further interesting implications for the optical spectra. In the following discussions, we present our findings regarding the linearly polarized optical response to normal incident light, obtained from the frequency dependent imaginary dielectric function $\left(\varepsilon_{2}(\omega)\right)$ and layer-dependent absorbance $(A(\omega))$ plots. The optical spectra of the hb and aw phases of SL Sb and Bi computed using the HSE + RPA approach are shown in Fig. 3 and 4. In this section we also highlight the effects of SOC on the optical response of $\mathrm{SL} \mathrm{Sb}$ and $\mathrm{Bi}$. We then extend our discussion by including the many-body effects. Due to the shortcomings of the standard DFT method for calculating the excited state properties, we do not include this approach in the discussion herewith.

As presented in Fig. 3(a), a main luminescence peak in the HSE + RPA in-plane optical response (i.e. light polarized along $x, y$ directions) for the hb-Sb phase is observed near $2.8 \mathrm{eV}$, where the onset is located slightly below $2 \mathrm{eV}$ and the range of this predominant absorption phenomenon is between $\sim 2.4-3.9 \mathrm{eV}$. The optical activity of hb-Sb continues further until $6 \mathrm{eV}$. Hence, the hb-phase of 2D Sb can be considered as a significant candidate SL material for devices operating under both visible and UV light. Regarding the out-of-plane response, i.e. light polarized along the $z$-axis (see Fig. 3(b)), a main single luminescence peak appears at $5.7 \mathrm{eV}$, thus indicating a 
Table 1 Structural parameters (lattice constants $a$ and $b$, bond lengths $d, d_{1}$ and $d_{2}$, and the buckling parameter $\Delta$, all calculated within LDA or PBE), and electronic band gaps of the $\mathrm{hb}$ and aw phases of antimonene and bismuthene, calculated from PBE-SOC, HSE, HSE-SOC and GoWo

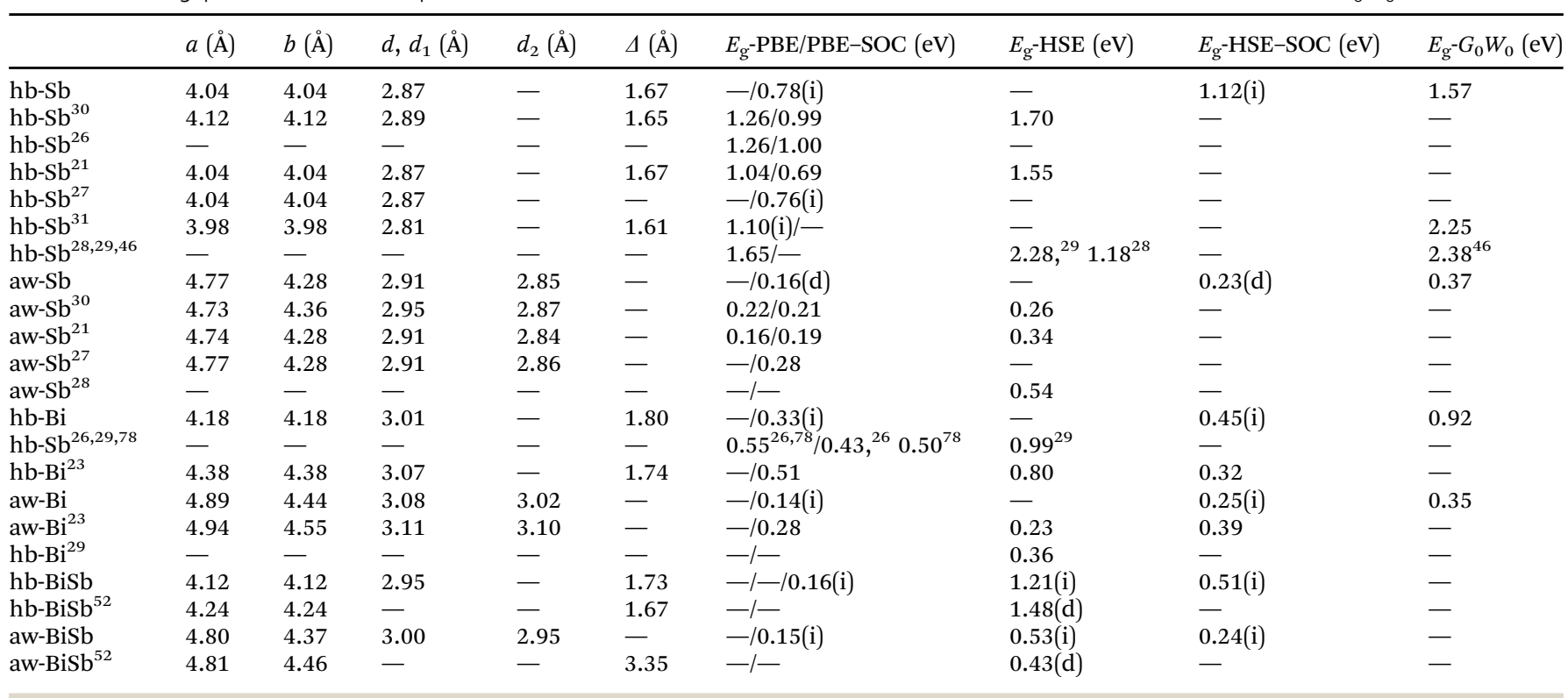
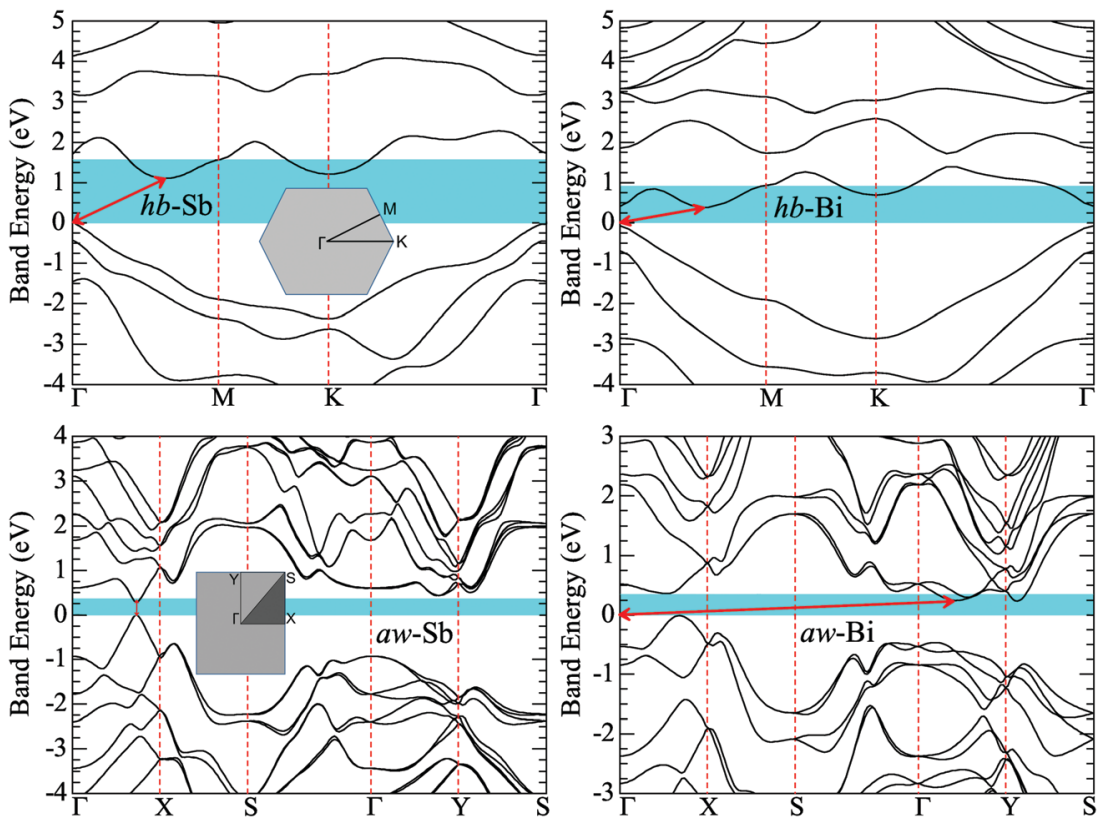

Fig. 2 The electronic band structure of SL hb-Sb, aw-Sb, hb-Bi and aw-Bi computed from HSE-SOC. GoW band gaps are shown by the shaded area. Red arrows indicate the fundamental band gaps. The zero energy is set at the top of the valence bands and Brillouin zones are shown by the insets.

different character of optical features with respect to the in-plane optical response.

The SOC effect on the optical properties of hb-Sb, as displayed in the $\varepsilon_{2}(\omega)$ and $A(\omega)$ plots in Fig. 3(a-c), is observed to be minor. Only a slight red-shift of the optical absorption band edge occurs once SOC is taken into account. The differences at the highest amplitude of the luminescence peaks arise from the computational artifacts. Referring to the HSE and HSE-SOC band gaps of hb-Sb near $1.2 \mathrm{eV}$, the minor onset shifts between both approaches are expected. The in-plane absorbance presented in the lower left panel shows that the main optical activity is concentrated between $2.5-6 \mathrm{eV}$. A further comment on a method-wise comparison, regarding the timedependent Hartree Fock approach to calculating the optical spectra, is that only minor differences from the HSE-RPA spectra of SL hb-, aw-Sb and hb-, aw-Bi structures were observed.

$\mathrm{SL}$ hb-Bi, with an isotropic in-plane optical response, also comes out as an interesting 2D material in terms of its linear optical properties. Although the absorption band edge is located slightly above $0.5 \mathrm{eV}$ (see the HSE + RPA spectrum in 

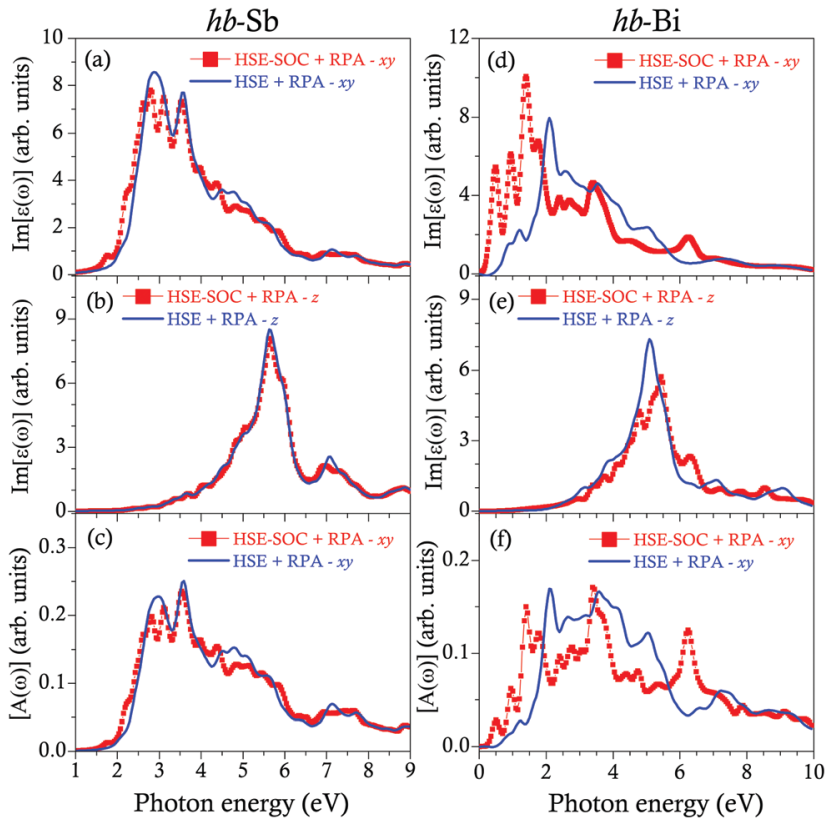

Fig. 3 Imaginary dielectric function $\left[\varepsilon_{2}(\omega)\right]$ and absorbance $[A(\omega)]$ as a function of the photon energy of hb-Sb computed from HSE + RPA and HSE-SOC + RPA: (a) for light polarized along $x$ and $y$ directions, and (b) along the $z$-direction. (c) Frequency dependent absorbance of hb-Sb for in-plane polarization. (d)-(f) Same as (a)-(c) calculated for hb-Bi.

Fig. 3(d)), the significant peak is set in the visible region, around $2.2 \mathrm{eV}$. However, due to bismuthene's well-known sensitivity, as can be observed from the HSE-SOC + RPA plot, both the onset and luminescence peak are red-shifted by nearly $0.5-0.6 \mathrm{eV}$ by incorporating the SOC effects. Once SOC is included in the HSE calculation, the $0.80 \mathrm{eV}$ HSE band gap is reduced to $0.32 \mathrm{eV}$, hence the onset and peak red-shift is in compliance with the significantly closed band gap of SL hb-Bi. The out-of-plane optical absorption yields highlighted peaking around $5.1 \mathrm{eV}$. As shown in Fig. 3(f), while the predominant absorbance revealed by HSE-SOC + RPA calculations is confined between $\sim 1.5-7 \mathrm{eV}$, without SOC effects the onset is delayed to $2 \mathrm{eV}$.

aw-Sb displays rather diversified optical features in comparison to hb-Sb. Owing to the narrow band gap of aw-Sb, no clear absorption band edge is observed in the $\varepsilon_{2}(\omega)$ especially for the HSE-SOC + RPA calculation, as evident in the left upper two panels of Fig. 4. A main difference from the hexagonal buckled phase's optical response is the anisotropy along the $x$ - and $y$-directions in the lattice. Although a kick-off in absorption occurs in the very early frequencies for both orientations, the onset is not as pronounced as in typical semiconductor photoluminescence behavior, which is even more evident in the HSESOC + RPA plot. The main peak in $\varepsilon_{2}(\omega)$ along $x$ is set at around $2.3 \mathrm{eV}$, with preliminary small peaks located at 0.4 and $1.2 \mathrm{eV}$, where the optical activity occurs up until $4 \mathrm{eV}$. The significant character regarding the optical properties of aw-Sb along $y$ is the well-pronounced range of absorption between 1.1-2.7 eV, indicating a rather continuous absorption with an onset in the IR, yet extending beyond the mid-visible light region. The effect
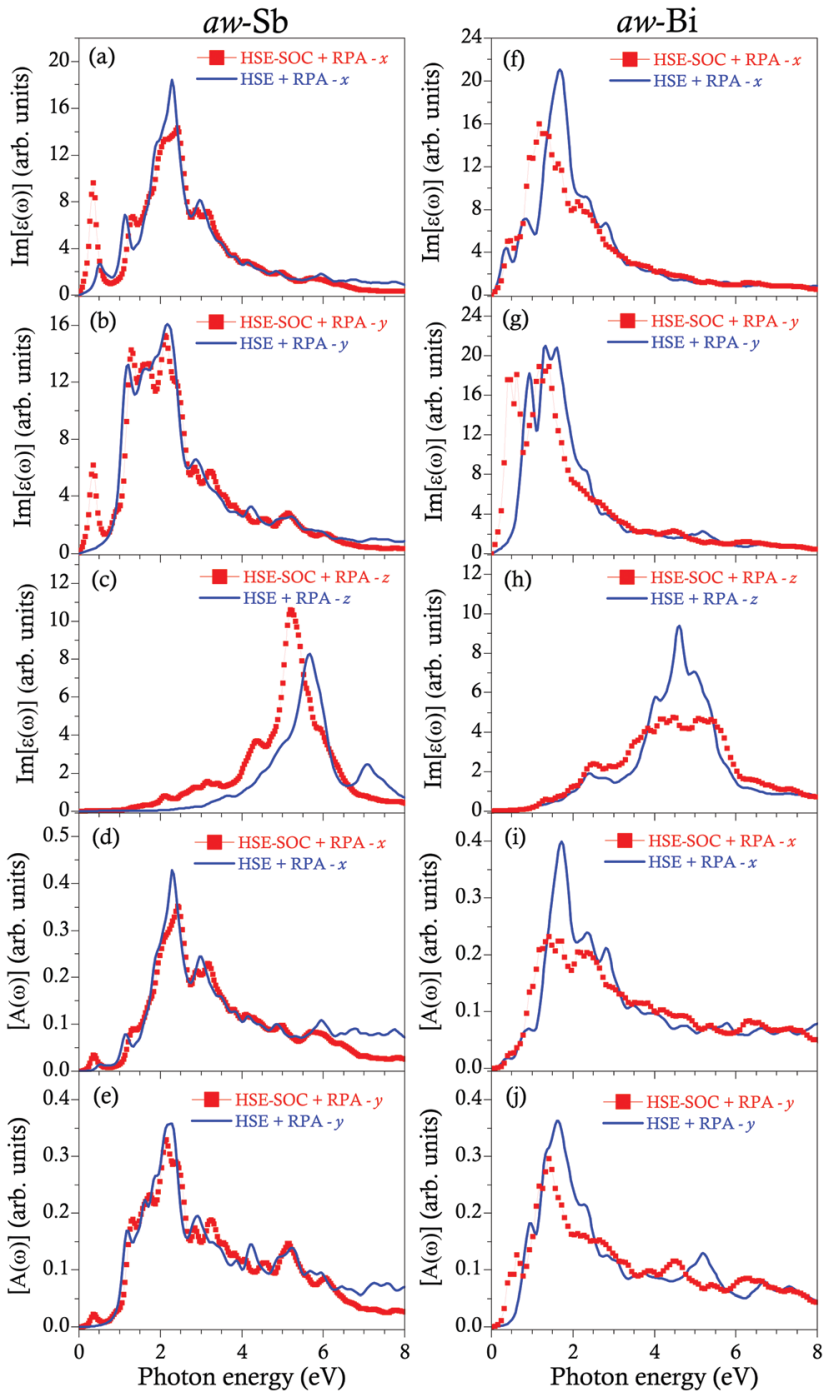

Fig. $4 \varepsilon_{2}(\omega)$ and $A(\omega)$ of aw-Sb computed as a function of photon energy from HSE + RPA and HSE-SOC + RPA: (a) $\varepsilon_{2}(\omega)$ for light polarized along the $x-$, (b) $y$-, and (c) $z$-directions. (d) $A(\omega)$ along $x$-, and (e) $y$-directions. (f)-(j) Same as (a)-(e) for aw-Bi.

of SOC on the in-plane optical response generally refers to an extra early smaller peak, without a major modification of the overall features. Also, according to the absorbance spectra (Fig. 4(d and e)), the optical features along $x$ and $y$ are very similar in the HSE and HSE-SOC calculations, mainly concentrated between $\sim 2-3.5 \mathrm{eV}$ for the former and $\sim 1.5-3 \mathrm{eV}$ for the latter. Finally, the out-of plane optical properties of aw-Sb indicate a strong absorption peak around $5.9 \mathrm{eV}$, which is red-shifted to $5.2 \mathrm{eV}$ by the SOC inclusion, and still situated in the UV range.

Similarly, the in-plane optical anisotropy within aw-Bi is also evident (see Fig. 4(f and g)). The HSE-SOC + RPA spectrum obtained for light polarized along $x$ is rather dispersed, with an onset in the vicinity of 0-limit and a shoulder feature near $0.9 \mathrm{eV}$. This is followed by the main peak located at $\sim 2.7 \mathrm{eV}$, and a broadened optical activity range until nearly $5 \mathrm{eV}$. On the other hand, the HSE-SOC + RPA spectrum along $y$ displays 
relatively concentrated and pronounced peaks within $\sim 0.4-2 \mathrm{eV}$. The SOC effects red-shift the optical spectra, due to the closing band gaps. The out-of-plane response displays that without SOC, a distinct peak is observed at $4.75 \mathrm{eV}$, however including SOC leads to a dispersed spectral distribution of absorption. Finally, as can be observed in Fig. 4(i and j), the anisotropic absorbance plots of aw-Bi show that the absorbance along $x$ is confined between $\sim 1-3.5 \mathrm{eV}$, and along $y$ within a slightly narrower scale; peaking roughly at $1.7 \mathrm{eV}$.

\section{Many-body optical properties of hb-Sb and hb-Bi}

Since $\mathrm{Sb}$ and $\mathrm{Bi}$ are group-VA elements with their $4 \mathrm{~d}$ and $5 \mathrm{~d}$ orbitals completely filled, respectively, many-body interactions are expected to be significant for both 2D structures, in terms of their electronic and optical properties. Hence, we extend our results in order to detect the impact of the many-body quasiparticle GW approach on the optical absorption spectra of two representative systems, hb-Sb and hb-Bi. On top of the corrected electronic energy states, i.e. the quasiparticle $G_{0} W_{0}$ energies, we account for the electron-hole interactions using the BSE, which are important due to the reduced dielectric screening based on the reduced dimensionality. We find that distinct excitonic peaks are obtained once strong direct transitions take place upon electron-hole interactions, disrupting the random phase of homogeneous transition matrix elements.

In Fig. 5 we present the optical absorption spectra of hb-Sb and hb-Bi computed from $G_{0} W_{0}+$ RPA, along with the $G_{0} W_{0}+$ BSE plots accounting for the excitonic effects. Also, the spectroscopic features regarding these spectra, such as the absorption onset and optical gap, are tabulated in Table 2. In the upper panel of Fig. 5, the light blue curve represents the $G_{0} W_{0}+$ RPA $\varepsilon_{2}(\omega)$ of hb-Sb. Accordingly, the absorption band edge corresponds to $\sim 2.4 \mathrm{eV}$. Beyond this onset, a relatively broad optical response is observed until $6-7 \mathrm{eV}$. The prominent spectral range of absorption is between $2.5-4 \mathrm{eV}$, the spectrum peaking at $2.88 \mathrm{eV}$, attributed to the interband transitions with resonant direct transition energies, covering partial visible and near UV light frequency ranges. On the other hand, the pink curve reveals a major excitonic peak located around $2.43 \mathrm{eV}$, with a narrow spectral width. Prior to the luminescence peak, an onset above $1.85 \mathrm{eV}$ appears. Since the minimum electronic band gap estimated by the $G_{0} W_{0}$ method for hb-Sb was found as $1.57 \mathrm{eV}$ (denoted by the vertical dashed blue line), we observe no exciton binding phenomenon according to our calculations. In Table 1 we also tabulate the results present in the literature where few previous studies ${ }^{31,46}$ have reported higher values of $G_{0} W_{0}$ gaps.

The lower panel of Fig. 5 displays the many-body optical response of the hb-Bi structure. Unlike hb-Sb, an absorption onset of the $G_{0} W_{0}+$ RPA plot occurs at lower photon energies, around $0.7 \mathrm{eV}$. As shown by the light blue curve, a small first peak is located at $\sim 0.9 \mathrm{eV}$, with optical activity extending beyond the visible light range. According to the $G_{0} W_{0}+\mathrm{BSE}$ results shown by the pink curve, with reference to the $G_{0} W_{0}$ band gap of hb-Bi at $0.92 \mathrm{eV}$, a low exciton binding energy of $\sim 0.18 \mathrm{eV}$ was found based on the highest peak amplitude at

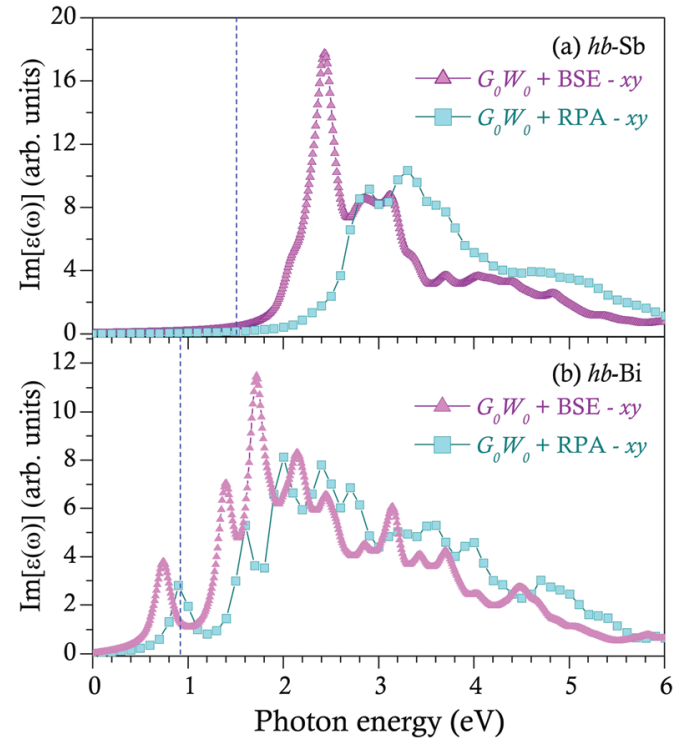

Fig. 5 (a) $\varepsilon_{2}(\omega)$ versus photon energy calculated for hb-Sb using $G_{0} W_{0}+$ RPA and $G_{0} W_{0}+$ BSE, the latter accounting for the excitonic effects for light polarized along the $x y$-directions. (b) Same as (a) for hb-Bi.

Table 2 The $G_{0} W_{0}$ band gap $\left(E_{\mathrm{g}}-G_{0} W_{0}\right), G_{0} W_{0}+$ RPA absorption spectrum onset (onset- $G_{0} W_{0}$ ) and peak position values (first one is denoted by peak$\left.G_{0} W_{0}\right), G_{0} W_{0}+$ BSE excitonic peak (i.e. the optical gap $E_{\text {opt }}$ ) and the exciton binding energy $\left(E_{\mathrm{b}}\right)$ values of pristine $\mathrm{hb} \mathrm{Sb}$ and $\mathrm{Bi}$. The peak positions subsequent to the first peak are given in parentheses in the rows below

\begin{tabular}{llllll}
\hline & $E_{\mathrm{g}}-G_{0} W_{0}$ & Onset- $G_{0} W_{0}$ & Peak- $G_{0} W_{0}$ & $E_{\text {opt }}$ & $E_{\mathrm{b}}$ \\
\hline $\mathrm{hb}-\mathrm{Sb}$ & 1.57 & 2.4 & $\begin{array}{l}2.88 \\
(3.3)\end{array}$ & $\begin{array}{l}2.43 \\
(2.85,3.12)\end{array}$ & - \\
& & & 0.9 & 0.74 & 0.18 \\
$\mathrm{hb}-\mathrm{Bi}$ & 0.92 & 0.7 & $(1.6,2.0)$ & $(1.4,1.72)$ &
\end{tabular}

All units are in eV.

$0.74 \mathrm{eV}$. Splitting of the excitonic peaks are observed, where we found evidence of only a first exciton binding. The onset of the first $G_{0} W_{0}+$ BSE peak is slightly above $\sim 0.5 \mathrm{eV}$, hence the offset of this onset from the electronic gap is around $0.4 \mathrm{eV}$. In comparison to buckled antimonene, the many-body optical response of buckled bismuthene is spectrally broader. In summary, our findings suggest that both SL hb-Sb and SL hb-Bi could be promising materials for future photovoltaic and other broadband optical applications.

\section{Electronic and optical properties of $\mathrm{hb}-\mathrm{BiSb}$ and aw-BiSb binary compound}

Having investigated the properties of pristine antimonene and bismuthene in detail, we turn our attention to the stable allotropes of SL BiSb binary compounds. ${ }^{34,35,44,52-56}$ Analogous to its pristine components, we focus on two different stable phases of the BiSb compound, namely the hb and aw allotropes. We investigate their electronic and optical properties using the HSE-SOC approach. The implications of forming a compound of 
$\mathrm{Sb}$ and $\mathrm{Bi}$ on the resulting optoelectronic properties could shed light on the increased functionalities of both materials.

The electronic band diagrams of the hb- and aw-BiSb binary compounds are displayed in Fig. 6. Both structures were found to be narrow and indirect band gap systems, the former phase with a HSE-SOC indirect band gap of 0.51 , and $0.24 \mathrm{eV}$ for the latter, both values much closer to that of pristine bismuthene rather than antimonene. The closing of the HSE gaps due to the SOC effects were found as 0.7 and $0.3 \mathrm{eV}$ for the hb-BiSb and aw-BiSb structures, respectively. Regarding the optical properties computed from HSE + RPA, as shown in Fig. 7, a sharp rise in the in-plane absorption of hb-BiSb occurs around $1.2 \mathrm{eV}$ with a luminescence peak located at $2.5 \mathrm{eV}$, and optical activity extending until nearly $5 \mathrm{eV}$ (see the uppermost panel). The absorption band edge, main peak positions and significant optical absorption range of hb-BiSb resemble those revealed by SL hb-Bi in comparison to hb-Sb, with an onset in the IR and main peak in the visible regimes. On the other hand, the out-ofplane optical response (see Fig. 7(b)) indicates main absorption peaking at $\sim 5.4 \mathrm{eV}$. When SOC effects are included, an extra, small early peak of the in-plane absorption appears at $0.8 \mathrm{eV}$, followed by a more distinct peak at $2.1 \mathrm{eV}$. In addition, a red-shift of the overall spectrum is observed owing to the closed band gap, once SOC effects are taken into account.

With regard to the optical response of aw-BiSb (see Fig. 7(c-e)) computed from HSE + RPA, a small shoulder after $0.5 \mathrm{eV}$ for light polarized along the $x$-direction, and a main peak with highest amplitude at nearly $2 \mathrm{eV}$ are observed. On the other hand, the HSE-SOC + RPA plot reveals an earlier narrow peak at $0.38 \mathrm{eV}$, and then a larger peak with the highest amplitude at $1.71 \mathrm{eV}$. The anisotropy within the HSE + RPA in-plane optical
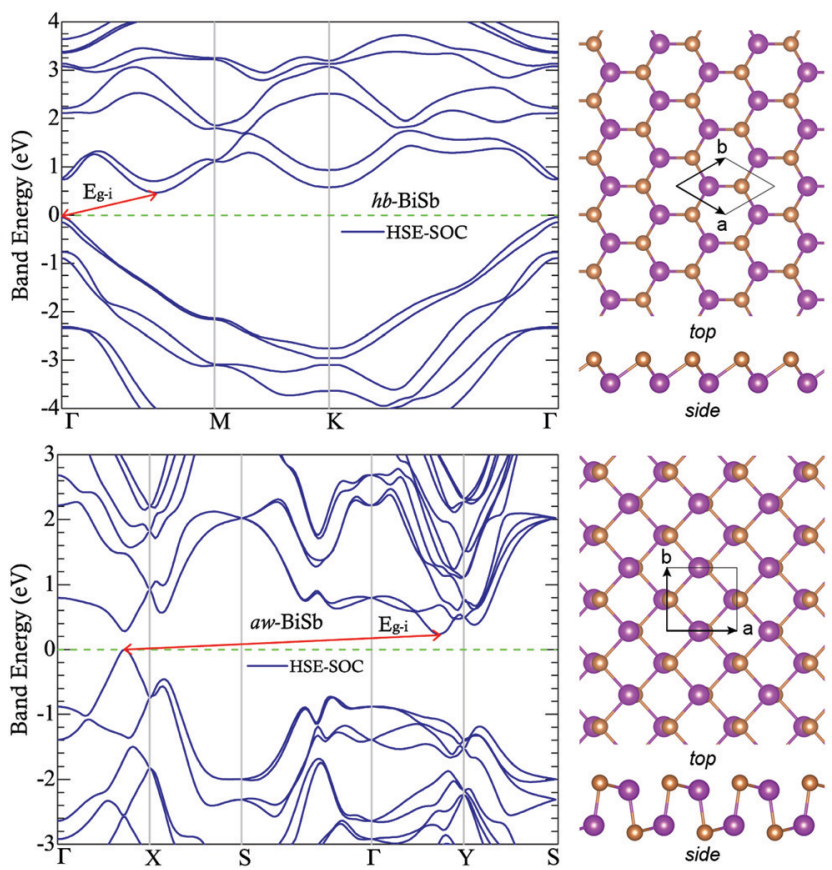

Fig. 6 The electronic energy band structures of the SL hb-BiSb and aw-BiSb binary compounds computed from HSE-SOC.

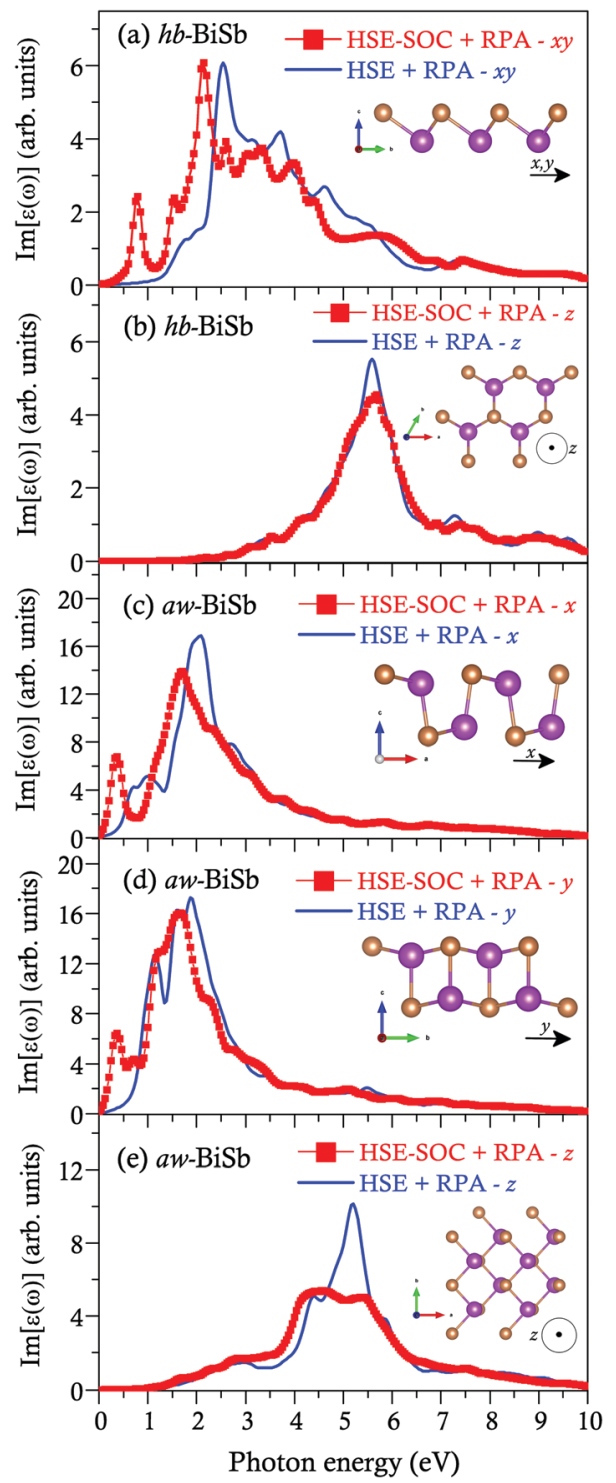

Fig. $7 \quad \varepsilon_{2}(\omega)$ as a function of photon energy of the hb-BiSb compound calculated from HSE + RPA and HSE-SOC + RPA, (a) for light polarized along the $x$ and $y$, and (b) $z$-directions. $\varepsilon_{2}(\omega)$ of the aw-BiSb compound calculated from HSE + RPA and HSE-SOC + RPA, for light polarized along the (c) $x-$, (d) $y$-, and (e) $z$-directions.

response is evident from the rather enlarged absorption peak along the $y$-direction, concentrated between $\sim 0.8-3 \mathrm{eV}$. The HSE-SOC + RPA spectrum along $y$ yields again an extra lowfrequency shoulder, along with a major absorption peak appearing at $1.67 \mathrm{eV}$. The optical activity is pursued until nearly $3 \mathrm{eV}$ for both orientations of the in-plane absorption. Hence, the BiSb compound in the asymmetric washboard structure displays significant optical features within the IR and visible regimes. Concerning the SOC effects, the global red-shift of $\varepsilon_{2}(\omega)$ is also valid for aw-BiSb, due to the reduced HSE-SOC band gap. Finally, the out-of-plane optical spectrum of aw-BiSb displays a distinct HSE + RPA peak at $\sim 5.2 \mathrm{eV}$, which is dispersed between 4.3-5.4 eV in the case of the HSE-SOC + RPA approach. 


\section{Discussions and conclusions}

In conclusion, we report that $2 \mathrm{D}$ antimonene and bismuthene structures with fundamental band gaps near the IR and visible light regions have promising optoelectronic properties for future broadband photonic and light modulator device applications. According to the HSE results, for hb-Sb, prominent optical absorption occurs within the visible regime with an onset of absorption at $\sim 2 \mathrm{eV}$ and within the IR for hexagonal buckled Bi. For both structures, the optical activity extends beyond the visible light range. On the other hand, for all other phases except $\mathrm{hb}-\mathrm{Sb}$, absorption begins to be active in the IR region. From the aspect of many-body effects, a systematic blue-shift of the spectral features from HSE-SOC + RPA to the quasiparticle $G_{0} W_{0}+$ RPA calculations is observed. A major excitonic peak for $\mathrm{hb}-\mathrm{Sb}$ is observed slightly above $2.4 \mathrm{eV}$, where no apparent exciton binding is evident. In summary, hb-Sb can be considered as a significant candidate SL material for devices operating under both visible and UV light. The SOC effects on the optical properties of hb-Sb are observed to be minor. Regarding the main HSE-SOC + RPA peak along the $\varepsilon_{2}(\omega)$ of aw-Sb, the prominent peak is set at around $2.3 \mathrm{eV}$, and the spectral features are active until $4 \mathrm{eV}$. Along the other in-plane crystalline orientation of aw-Sb, the wellpronounced range of absorption is between 1.1-2.7 eV, indicating a rather continuous absorption with an onset in the IR, and extending beyond the mid-visible light region. In view of effective solar absorption, antimonene stands out with clear luminescence peaks concentrated in the visible range.

We also show that inclusion of spin-orbit coupling effects modifies the optical spectra of bismuthene to a significant extent. Although the absorption onset of hb-Bi is located slightly above $0.5 \mathrm{eV}$, the major peak of absorption is set in the visible frequencies, around $2.2 \mathrm{eV}$. On the other hand, both the absorption onset and luminescence peak are red-shifted by $\sim 0.6 \mathrm{eV}$, when SOC effects are included. The in-plane optical response of hb-Bi is substantially affected once the electronhole interactions are taken into account, with enhanced absorption even above the quasiparticle gap. A first exciton binding with $0.18 \mathrm{eV}$ binding energy is predicted. The anisotropic optical absorbance of aw-Bi is rather confined between $\sim 1-3.5 \mathrm{eV}$ along the $x$-direction, and within a narrower scale along $y$, peaking at $\sim 1.7 \mathrm{eV}$. Similarly, SOC leads to a red-shift of the optical spectra of aw-Bi, owing to the reduced band gap values. In general, the differences in the optical spectra caused by the SOC effects are more distinguished for the Bi phases compared to $\mathrm{Sb}$. Moreover, the in-plane optical response is modified upon including the SOC effects, in a more obvious way in comparison to the out-of-plane direction. However, no clear trends regarding the differences in the optical response along the $x$ or $y$-directions were observed.

The optical properties of the SL BiSb structures can either be isotropic or anisotropic, with the possibility of tuning the optoelectronic properties based on the stoichiometric mixing proportion of $\mathrm{Sb}$ and $\mathrm{Bi}$ atoms. Due to the small band gaps of the SL BiSb compound, the HSE-SOC + RPA calculations indicate early in-plane absorption band edges for both structures.
Both the hb- and aw- phases reveal optical activities spanning the visible regime, and partially extending to the UV range.

In summary, the electronic band gap, optical gap and exciton binding energies can be tuned depending on the different crystalline orientations, which could be useful for light manipulation or modulation. Furthermore, SOC effects impose different levels of alterations in the optoelectronic properties of SL pristine Sb, Bi and the binary compound BiSb. While we find an exciton binding phenomenon for the buckled phase of bismuthene, many-body effects are still prominent regarding the optoelectronic properties of $2 \mathrm{D} \mathrm{Sb}, \mathrm{Bi}$ and $\mathrm{BiSb}$. We hope that this study will contribute to the knowledge necessary for next-generation devices based on $2 \mathrm{D} \mathrm{Sb}$, Bi and the BiSb compound, and for exploring new physics in ultrathin group-VA members.

\section{Conflicts of interest}

There are no conflicts to declare.

\section{Acknowledgements}

The calculations were performed at TUBITAK ULAKBIM, High Performance and Grid Computing Center (TR-Grid e-Infrastructure) and the National Center for High Performance Computing of Turkey (UHeM) under grant no. 5003622015. This work was supported by the Scientific and Technological Research Council of Turkey (TUBITAK) under Project No. 115F088. E. D. acknowledges support from The Turkish Academy of Sciences - Outstanding Young Scientists Award Program (TUBA-GEBIP). S. C. acknowledges the financial support from Academy of Science of Turkey (TÜBA).

\section{References}

1 K. S. Novoselov, A. K. Geim, S. V. Morozov, D. Jiang, Y. Zhang, S. V. Dubonos, I. V. Grigorieva and A. A. Firsov, Electric Field Effect in Atomically Thin Carbon Films, Science, 2004, 306, 666-669.

2 J. N. Coleman, M. Lotya, A. O’Neill, S. D. Bergin, P. J. King, U. Khan, K. Young, A. Gaucher, S. De and R. J. Smith, et al., Two-dimensional Nanosheets Produced by Liquid Exfoliation of Layered Materials, Science, 2011, 331, 568-571.

3 K. Novoselov, D. Jiang, F. Schedin, T. Booth, V. Khotkevich, S. Morozov and A. Geim, Two-dimensional Atomic Crystals, Proc. Natl. Acad. Sci. U. S. A., 2005, 102, 10451-10453.

4 C. Ataca and S. Ciraci, Functionalization of Single-layer $\mathrm{MoS}_{2}$ Honeycomb Structures, J. Phys. Chem. C, 2011, 115, 13303-13311.

5 C. Ataca, H. Sahin and S. Ciraci, Stable, Single-layer $\mathbf{M X}_{2}$ Transition-metal Oxides and Dichalcogenides in a Honeycomb-like Structure, J. Phys. Chem. C, 2012, 116, 8983-8999.

6 V. O. Özçelik, M. Fathi, J. G. Azadani and T. Low, Tin Monochalcogenide Heterostructures as Mechanically Rigid 
Infrared Band Gap Semiconductors, Phys. Rev. Mater., 2018, 2, 051003.

7 E. Durgun, S. Tongay and S. Ciraci, Silicon and III-V Compound Nanotubes: Structural and Electronic Properties, Phys. Rev. B: Condens. Matter Mater. Phys., 2005, 72, 075420.

8 K. K. Kim, A. Hsu, X. Jia, S. M. Kim, Y. Shi, M. Hofmann, D. Nezich, J. F. Rodriguez-Nieva, M. Dresselhaus and T. Palacios, et al., Synthesis of Monolayer Hexagonal Boron Nitride on $\mathrm{Cu}$ Foil Using Chemical Vapor Deposition, Nano Lett., 2012, 12, 161-166.

9 S. Cahangirov, M. Topsakal and S. Ciraci, Armchair Nanoribbons of Silicon and Germanium Honeycomb Structures, Phys. Rev. B: Condens. Matter Mater. Phys., 2010, 81, 195120.

10 H. Sahin, S. Cahangirov, M. Topsakal, E. Bekaroglu, E. Akturk, R. T. Senger and S. Ciraci, Monolayer Honeycomb Structures of Group-IV Elements and III-V Binary Compounds: First-principles Calculations, Phys. Rev. B: Condens. Matter Mater. Phys., 2009, 80, 155453.

11 D. Kecik, A. Onen, M. Konuk, E. Gürbüz, F. Ersan, S. Cahangirov, E. Aktürk, E. Durgun and S. Ciraci, Fundamentals, Progress, and Future Directions of Nitride-based Semiconductors and Their Composites in Two-dimensional Limit: A First-principles Perspective to Recent Synthesis, Appl. Phys. Rev., 2018, 5, 011105.

12 A. Du, In silico engineering of graphene-based van der Waals heterostructured nanohybrids for electronics and energy applications, Wiley Interdiscip. Rev.: Comput. Mol. Sci., 2016, 6, 551-570.

13 X. Zhang, Z. Meng, D. Rao, Y. Wang, Q. Shi, Y. Liu, H. Wu, K. Deng, H. Liu and R. Lu, Efficient band structure tuning, charge separation, and visible-light response in $\mathrm{ZrS}_{2}$-based van der Waals heterostructures, Energy Environ. Sci., 2016, 9, 841-849.

14 P. Wang, S. Liu, W. Luo, H. Fang, F. Gong, N. Guo, Z.-G. Chen, J. Zou, Y. Huang and X. Zhou, et al., Arrayed van der Waals Broadband Detectors for Dual-Band Detection, Adv. Mater., 2017, 29, 1604439.

15 L. Li, Y. Yu, G. J. Ye, Q. Ge, X. Ou, H. Wu, D. Feng, X. H. Chen and Y. Zhang, Black Phosphorus Field-effect Transistors, Nat. Nanotechnol., 2014, 9, 372.

16 V. O. Özcelik, O. Ü. Aktürk, E. Durgun and S. Ciraci, Prediction of a Two-dimensional Crystalline Structure of Nitrogen Atoms, Phys. Rev. B: Condens. Matter Mater. Phys., 2015, 92, 125420.

17 H. Liu, A. T. Neal, Z. Zhu, Z. Luo, X. Xu, D. Tománek and P. D. Ye, Phosphorene: An Unexplored 2D Semiconductor with a High Hole Mobility, ACS Nano, 2014, 8, 4033-4041.

18 C. Kamal and M. Ezawa, Arsenene: Two-dimensional Buckled and Puckered Honeycomb Arsenic Systems, Phys. Rev. B: Condens. Matter Mater. Phys., 2015, 91, 085423.

19 D. Kecik, E. Durgun and S. Ciraci, Stability of Single-layer and Multilayer Arsenene and Their Mechanical and Electronic Properties, Phys. Rev. B, 2016, 94, 205409.

20 F. Ersan, E. Aktürk and S. Ciraci, Stable Single-layer Structure of Group-V Elements, Phys. Rev. B, 2016, 94, 245417.
21 O. Ü. Aktürk, V. O. Özçelik and S. Ciraci, Single-layer Crystalline Phases of Antimony: Antimonenes, Phys. Rev. B: Condens. Matter Mater. Phys., 2015, 91, 235446.

22 S. Zhang, Z. Yan, Y. Li, Z. Chen and H. Zeng, Atomically Thin Arsenene and Antimonene: Semimetal-semiconductor and Indirect-direct Band-gap Transitions, Angew. Chem., Int. Ed., 2015, 54, 3112-3115.

23 E. Aktürk, O. Ü. Aktürk and S. Ciraci, Single and Bilayer Bismuthene: Stability at High Temperature and Mechanical and Electronic Properties, Phys. Rev. B, 2016, 94, 014115.

24 R. R. Freitas, R. Rivelino, F. de Brito Mota, C. de Castilho, A. Kakanakova-Georgieva and G. K. Gueorguiev, Topological Insulating Phases in Two-dimensional Bismuth-containing Single Layers Preserved by Hydrogenation, J. Phys. Chem. C, 2015, 119, 23599-23606.

25 S. Zhang, S. Guo, Z. Chen, Y. Wang, H. Gao, J. GómezHerrero, P. Ares, F. Zamora, Z. Zhu and H. Zeng, Recent Progress in 2D Group-VA Semiconductors: From Theory to Experiment, Chem. Soc. Rev., 2018, 47, 982-1021.

26 J. Lee, W.-C. Tian, W.-L. Wang and D.-X. Yao, Twodimensional Pnictogen Honeycomb Lattice: Structure, on-site Spin-orbit Coupling and Spin Polarization, Sci. Rep., 2015, 5, 11512.

27 G. Wang, R. Pandey and S. P. Karna, Atomically Thin Group V Elemental Films: Theoretical Investigations of Antimonene Allotropes, ACS Appl. Mater. Interfaces, 2015, 7, 11490-11496.

28 D. Singh, S. K. Gupta, Y. Sonvane and I. Lukačević, Antimonene: A Monolayer Material for Ultraviolet Optical Nanodevices, J. Mater. Chem. C, 2016, 4, 6386-6390.

29 S. Zhang, M. Xie, F. Li, Z. Yan, Y. Li, E. Kan, W. Liu, Z. Chen and H. Zeng, Semiconducting Group 15 Monolayers: a Broad Range of Band Gaps and High Carrier Mobilities, Angew. Chem., 2016, 128, 1698-1701.

30 Y. Xu, B. Peng, H. Zhang, H. Shao, R. Zhang and H. Zhu, First-principle Calculations of Optical Properties of Monolayer Arsenene and Antimonene Allotropes, Ann. Phys, 2017, 529, 1600152.

31 H. Shu, Y. Li, X. Niu and J. Guo, Electronic Structures and Optical Properties of Arsenene and Antimonene Under Strain and an Electric Field, J. Mater. Chem. C, 2018, 6, 83-90.

32 Y. Luo, Y. Li, P. Guo, W. Chen, Y. Tang and Y. Jia, Band Structures of One-dimensional Buckled Arsenene Nanoribbons: Strain and Quantum Size Modulations, Mod. Phys. Lett. B, 2017, 31, 1750341.

33 Y. Wang and Y. Ding, Electronic Structure and Carrier Mobilities of Arsenene and Antimonene Nanoribbons: A First-principle Study, Nanoscale Res. Lett., 2015, 10, 254.

34 S. Singh and A. H. Romero, Giant Tunable Rashba Spin Splitting in a Two-dimensional BiSb Monolayer and in BiSb/AlN Heterostructures, Phys. Rev. B, 2017, 95, 165444.

35 W. Yu, C.-Y. Niu, Z. Zhu, X. Cai, L. Zhang, S. Bai, R. Zhao and Y. Jia, Strain Induced Quantum Spin Hall Insulator in Monolayer $\beta$-BiSb from First-principles Study, RSC Adv., 2017, 7, 27816-27822. 
36 I. Aguilera, C. Friedrich and S. Blügel, Electronic Phase Transitions of Bismuth Under Strain from Relativistic Selfconsistent GW Calculations, Phys. Rev. B: Condens. Matter Mater. Phys., 2015, 91, 125129.

37 Y. Kadioglu, S. B. Kilic, S. Demirci, O. Ü. Aktürk, E. Aktürk and S. Ciraci, Modification of Electronic Structure, Magnetic Structure, and Topological Phase of Bismuthene by Point Defects, Phys. Rev. B, 2017, 96, 245424.

38 T. Märkl, P. J. Kowalczyk, M. Le Ster, I. V. Mahajan, H. Pirie, Z. Ahmed, G. Bian, X. Wang, T.-C. Chiang and S. A. Brown, Engineering Multiple Topological Phases in Nanoscale van der Waals Heterostructures: Realisation of $\alpha$-antimonene, 2 D Mater., 2017, 5, 011002.

39 Y. Shao, Z.-L. Liu, C. Cheng, X. Wu, H. Liu, C. Liu, J.-O. Wang, S.-Y. Zhu, Y.-Q. Wang and D.-X. Shi, et al., Epitaxial Growth of Flat Antimonene Monolayer: A New Honeycomb Analogue of Graphene, Nano Lett., 2018, 18, 2133-2139.

40 H.-S. Tsai, C.-W. Chen, C.-H. Hsiao, H. Ouyang and J.-H. Liang, The Advent of Multilayer Antimonene Nanoribbons with Room Temperature Orange Light Emission, Chem. Commun., 2016, 52, 8409-8412.

41 F. Reis, G. Li, L. Dudy, M. Bauernfeind, S. Glass, W. Hanke, R. Thomale, J. Schäfer and R. Claessen, Bismuthene on a SiC Substrate: A Candidate for a High-temperature Quantum Spin Hall Material, Science, 2017, 357, 287-290.

42 A. Zunger, Inverse Design in Search of Materials with Target Functionalities, Nat. Rev. Chem., 2018, 2, 0121.

43 P. Cucka and C. Barrett, The Crystal Structure of Bi and of Solid Solutions of $\mathrm{Pb}, \mathrm{Sn}, \mathrm{Sb}$ and Te in Bi, Acta Crystallogr., 1962, 15, 865-872.

44 S. Singh, W. Ibarra-Hernández, I. Valencia-Jaime, G. AvendañoFranco and A. H. Romero, Investigation of Novel Crystal Structures of Bi-Sb Binaries Predicted Using the Minima Hopping Method, Phys. Chem. Chem. Phys., 2016, 18, 29771-29785.

45 F. Xia, S. Xiong, Y. He, Z. Shao, X. Zhang and J. Jie, Tuning the Electronic and Optical Properties of Monolayers As, Sb, and Bi via Surface Charge Transfer Doping, J. Phys. Chem. C, 2017, 121, 19530-19537.

46 Y. Wang, P. Huang, M. Ye, R. Quhe, Y. Pan, H. Zhang, H. Zhong, J. Shi and J. Lu, Many-body Effect, Carrier Mobility, and Device Performance of Hexagonal Arsenene and Antimonene, Chem. Mater., 2017, 29, 2191-2201.

47 F. Chu, M. Chen, Y. Wang, Y. Xie, B. Liu, Y. Yang, X. An and Y. Zhang, A Highly Polarization Sensitive Antimonene Photodetector with a Broadband Photoresponse and Strong Anisotropy, J. Mater. Chem. C, 2018, 6, 2509-2514.

48 G. Wang, S. Higgins, K. Wang, D. Bennett, N. Milosavljevic, J. J. Magan, S. Zhang, X. Zhang, J. Wang and W. J. Blau, Intensity-dependent Nonlinear Refraction of Antimonene Dispersions in the Visible and Near-infrared Region, Appl. Opt., 2018, 57, E147-E153.

49 R.-B. Chen, D.-J. Jang, M.-C. Lin and M.-F. Lin, Optical Properties of Monolayer Bismuthene in Electric Fields, arXiv:1803.04649, 2018.
50 L. Lu, Z. Liang, L. Wu, Y. Chen, Y. Song, S. C. Dhanabalan, J. S. Ponraj, B. Dong, Y. Xiang and F. Xing, et al., Few-layer Bismuthene: Sonochemical Exfoliation, Nonlinear Optics and Applications for Ultrafast Photonics with Enhanced Stability, Laser Photonics Rev., 2018, 12, 1700221.

51 H. Huang, X. Ren, Z. Li, H. Wang, Z. Huang, H. Qiao, P. Tang, J. Zhao, W. Liang and Y. Ge, et al., Two-dimensional Bismuth Nanosheets as Prospective Photo-detector with Tunable Optoelectronic Performance, Nanotechnology, 2018, 29, 235201.

52 W.-Z. Xiao, G. Xiao, Q.-Y. Rong and L.-L. Wang, New Twodimensional VV Binary Compounds with a Honeycomb-like Structure: A First-principles Study, Mater. Res. Express, 2018, 5, 035903.

53 S. B. Pillai, S. D. Dabhi, S. Narayan and P. K. Jha, Strain Effect on Electronic and Lattice Dynamical Behaviour of Two dimensional Bi, BiAs and BiSb, AIP Conf. Proc., 2018, 090022.

54 W.-X. Ji, C.-W. Zhang, M. Ding, P. Li and P.-J. Wang, Quantum Spin Hall Phase Transitions in Two-dimensional SbBi Alloy Films, J. Mater. Chem. C, 2017, 5, 2649-2655.

55 S. Singh, I. Valencia-Jaime, O. Pavlic and A. H. Romero, Elastic, Mechanical, and Thermodynamic Properties of BiSb Binaries: Effect of Spin-orbit Coupling, Phys. Rev. B, 2018, 97, 054108.

56 C. Tan, P. Zhou, J. Lin and L. Sun, Prediction of Twodimensional BiSb with Puckered Structure, Phys. Status Solidi RRL, 2017, 11, 1700051.

57 P. E. Blöchl, Projector Augmented-wave Method, Phys. Rev. B: Condens. Matter Mater. Phys., 1994, 50, 17953.

58 G. Kresse and D. Joubert, From Ultrasoft Pseudopotentials to the Projector Augmented-wave Method, Phys. Rev. B: Condens. Matter Mater. Phys., 1999, 59, 1758.

59 M. Gajdoš, K. Hummer, G. Kresse, J. Furthmüller and F. Bechstedt, Linear Optical Properties in the Projectoraugmented Wave Methodology, Phys. Rev. B: Condens. Matter Mater. Phys., 2006, 73, 045112.

60 G. Kresse and J. Hafner, $A b$ initio Molecular-dynamics Simulation of the Liquid-metal-amorphous-semiconductor Transition in Germanium, Phys. Rev. B: Condens. Matter Mater. Phys., 1994, 49, 14251.

61 G. Kresse and J. Furthmüller, Efficient Iterative Schemes for $A b$ initio Total-energy Calculations Using a Plane-wave Basis set, Phys. Rev. B: Condens. Matter Mater. Phys., 1996, 54, 11169-11186.

62 J. P. Perdew, K. Burke and M. Ernzerhof, Generalized Gradient Approximation Made Simple, Phys. Rev. Lett., 1996, 77, 3865.

63 J. Paier, M. Marsman, K. Hummer, G. Kresse, I. C. Gerber and J. G. Ángyán, Screened Hybrid Density Functionals Applied to Solids, J. Chem. Phys., 2006, 124, 154709.

64 J. Heyd, G. E. Scuseria and M. Ernzerhof, Hybrid Functionals Based on a Screened Coulomb Potential, J. Chem. Phys., 2003, 118, 8207-8215.

65 J. Heyd and G. E. Scuseria, Efficient Hybrid Density Functional Calculations in Solids: Assessment of the Heyd-ScuseriaErnzerhof Screened Coulomb Hybrid Functional, J. Chem. Phys., 2004, 121, 1187-1192. 
66 L. Hedin, New Method for Calculating the One-particle Green's Function with Application to the Electron-gas Problem, Phys. Rev., 1965, 139, A796.

67 M. S. Hybertsen and S. G. Louie, Electron Correlation in Semiconductors and Insulators: Band Gaps and Quasiparticle Energies, Phys. Rev. B: Condens. Matter Mater. Phys., 1986, 34, 5390.

68 M. Shishkin and G. Kresse, Implementation and Performance of the Frequency-dependent GW Method within the PAW Framework, Phys. Rev. B: Condens. Matter Mater. Phys., 2006, 74, 035101.

69 D. Pines and D. Bohm, A Collective Description of Electron Interactions: II. Collective vs Individual Particle Aspects of the Interactions, Phys. Rev., 1952, 85, 338.

70 H. Ehrenreich and M. Cohen, Self-Consistent Field Approach to the Many-Electron Problem, Phys. Rev., 1959, 115, 786.

71 S. Steiner, S. Khmelevskyi, M. Marsmann and G. Kresse, Calculation of the magnetic anisotropy with projectedaugmented-wave methodology and the case study of disordered $\mathrm{Fe}_{1-x} \mathrm{Co}_{x}$ alloys, Phys. Rev. B, 2016, 93, 224425.
72 E. E. Salpeter and H. A. Bethe, A Relativistic Equation for Bound-state Problems, Phys. Rev., 1951, 84, 1232.

73 S. Albrecht, L. Reining, R. Del Sole and G. Onida, Ab initio Calculation of Excitonic Effects in the Optical Spectra of Semiconductors, Phys. Rev. Lett., 1998, 80, 4510.

74 M. Rohlfing and S. G. Louie, Electron-hole Excitations in Semiconductors and Insulators, Phys. Rev. Lett., 1998, 81, 2312.

75 L. X. Benedict, E. L. Shirley and R. B. Bohn, Optical Absorption of Insulators and the Electron-hole Interaction: An Ab initio Calculation, Phys. Rev. Lett., 1998, 80, 4514.

76 O. Ü. Aktürk, E. Aktürk and S. Ciraci, Effects of Adatoms and Physisorbed Molecules on the Physical Properties of Antimonene, Phys. Rev. B, 2016, 93, 035450.

77 P. Ares, J. J. Palacios, G. Abellán, J. Gómez-Herrero and F. Zamora, Recent Progress on Antimonene: A New Bidimensional Material, Adv. Mater., 2018, 30, 1-27.

78 L. Cheng, H. Liu, X. Tan, J. Zhang, J. Wei, H. Lv, J. Shi and $\mathrm{X}$. Tang, Thermoelectric Properties of a Monolayer Bismuth, J. Phys. Chem. C, 2013, 118, 904-910. 